\title{
Epidemiological Implications of HIV-Hepatitis C Co-Infection in South and Southeast Asia
}

\author{
Shaodong Ye • Lin Pang $•$ Xiaochun Wang $\cdot$ Zhongfu Liu
}

Published online: 29 March 2014

(C) The Author(s) 2014. This article is published with open access at Springerlink.com

\begin{abstract}
We sought to profile the epidemiological implication of human immunodeficiency virus (HIV) and hepatitis C virus $(\mathrm{HCV})$ co-infection from South and Southeast Asia by reviewing original studies reporting prevalence of HIV-HCV co-infection and their risk factors. Thirteen papers cited in the PubMed database and published in 2012 and 2013 were reviewed. The overall $\mathrm{HCV}$ co-infection prevalence ranged broadly from $1.2 \%$ to $98.5 \%$ among HIV-positive people in South and Southeast Asia. Among HCV seropositive blood donors in Nepal, $5.75 \%$ had HIV co-infection. Injecting drug use (IDU) was one of the key risk factors of co-infection, with HCV infection reaching $89.8 \%$ and $98.5 \%$ among HIVpositive injecting drug users in Vietnam. The most recent data from South and Southeast Asia suggest the urgency of implementation of comprehensive prevention and control strategies of HIV-HCV co-infection.
\end{abstract}

Keywords HIV · Hepatitis C · HIV-HCV Co-infection · Risk factors $\cdot$ South and Southeast Asia $\cdot$ Injection drug use $\cdot$ Blood donor $\cdot$ Prevalence $\cdot$ Global epidemic $\cdot$ HIV-positive $\cdot$

Southeast Asia $\cdot$ South Asia

\section{Introduction}

The 150 million people chronically infected with hepatitis C virus (HCV) suggest a $2.3 \%$ global prevalence, higher even than the 35.3 million people living with human immunodeficiency virus (HIV), about $0.54 \%$ prevalence. However, death rates are inverted with about 350,000 deaths/year from HCV-

S. Ye $(\bowtie) \cdot$ L. Pang $\cdot$ X. Wang $\cdot$ Z. Liu

National Center for AIDS/STD Control and Prevention, China

Center for Disease Control and Prevention, 155 Changbai Road,

Changping District, Beijing 102206, People's Republic of China

e-mail: yeshaodong@chinaaids.cn related liver diseases to 1.6 million deaths/year due to HIV/ AIDS, as estimated in 2013 by the World Health Organization (WHO) and the Joint United Nations Program on HIV/AIDS (UNAIDS) $[1 \cdot 2 \bullet]$.

Given sexual, blood, and perinatal transmission routes, HIV and HCV can be expected to be transmitted in similar at-risk populations. Reports are variable, but reports range from 7-30 \% HCV co-infection among HIV patients [3-7] and $6-10 \%$ HIV co-infection among HCV patients $[5,8 \bullet \bullet$. Co-infections influence each other and affect the pathogenesis and even causes of death for HIV-HCV infected persons, complicating both treatment and even prevention strategies $[9,10]$.

The estimated number of HIV cases in 19 countries of South and Southeast Asia ranks second just after SubSaharan Africa [2•]. Many of these nations have made substantial efforts to combat HIV/AIDS with a variety of education and prevention strategies, including efforts towards stigma reduction, interventions targeting commercial sex workers (CSW), injection drug users (IDU), expanded antiretroviral treatment (ART), and ensuring a safe blood supply, among others.

$\mathrm{HCV}$ infections are serious in these same countries, too, due to blood transfusions and IDU $[11,12]$. With the expansion of the HIV epidemic, increased awareness, and better diagnostic capacities, more HIV-HCV co-infections have been reported with increasing frequency $[11,13-15,16 \bullet$, $17 \bullet, 18 \bullet \cdot$. Many studies have introduced these coepidemics, analyzed the risk factors, discussed treatment, and suggested strategies of prevention and control in individual countries. While a few reviews included South and Southeast Asia [19, 20], there has been little targeted attention on HIV-HCV co-infection specifically for South and Southeast Asia.

Our paper aims uses recently published literature to provide a profile of the epidemiological circumstances for HIV-HCV 
prevalence and risk factors in South and Southeast Asia. We hope this stimulates increased attention to both prevention and clinical care for these highly populous nations.

\section{Methods}

Search Strategy The literature on HIV-HCV co-infection published in English in 2012 and 2013 was searched with the PubMed database. Keywords used in the database search included: [(hepatitis C or HCV) AND (HIV OR AIDS) AND (2012 OR 2013)] AND [(South Asia or Southeast Asia) or (individual country names of South and Southeast Asia)] (Table 1): All publications were sorted with an Endnote $^{\circledR}$ file (Endnote $X 4^{\circledR}$, Thomson Reuters, San Francisco, CA: Full texts were obtained from ProQuest ${ }^{\circledR}$ Health \& Medical through the Chinese Center for Disease Control and Prevention.

Study Selection The following criteria were used to select studies: original study on HIV-HCV co-infection; available prevalence rate of co-infection and/or odds ratio (OR) for putative risk factors; eligible countries of South and Southeast Asia. All titles and abstracts were reviewed to decide the inclusion of the eligible papers. Full texts were also reviewed whenever necessary, to check the data details and to judge the relevance of the epidemiological observations of HIV-HCV co-infection.

\section{Results}

Result of Literature Search A total of 23 and 39 papers were found in South and Southeast Asia in 2012 and 2013, respectively, of which 54 were unique (8 duplicates). A further 10 papers were excluded because they were not actually studies in South and Southeast Asia. Nine more papers were excluded due to containing only HIV or HCV information. Reviews of the abstracts and full texts of the remaining 35 papers led to further exclusion of 22 papers which had no data regarding HIV-HCV co-infection. Thus a total of 13 papers met the review needs $[8 \cdot \bullet, 16 \bullet \bullet, 17 \bullet \bullet, 18 \bullet \bullet, 21 \bullet \bullet, 22 \bullet \bullet, 23 \bullet \bullet, 24 \bullet$, $25 \bullet \bullet, 26 \bullet \bullet, 27 \bullet \bullet, 28 \bullet \bullet, 29 \bullet \cdot]$.

Description of Studies Among the 13 studies shown in Table 2, two South Asian studies were conducted in India and three in Nepal. Among eight Southeast Asian studies, two were in Vietnam, one in Cambodia, one in Myanmar, two in Thailand, and two in Indonesia. Except for one retrospective cohort and one case control study, 11 were designed as crosssectional studies. The sample size of the studies ranged widely from 126 to 16,124. Eight studies used HIV-positive samples
Table 1 Countries of South and Southeast Asia included in this literature-based review

\begin{tabular}{ll}
\hline South Asian countries & Southeast Asian countries \\
\hline - Bangladesh & • Brunei \\
- Pakistan & - Thailand \\
- Nepal & - East Timor \\
- Maldives & - Vietnam \\
- Republic of India & - Indonesia \\
- Sri Lanka & - Malaysia \\
- Bhutan & - Philippines \\
- Afghanistan & - Myanmar \\
& - Cambodia \\
& - Singapore \\
\hline
\end{tabular}

to assess HCV prevalence including one study among HIVinfected IDUs. Four studies used high risk populations, including one study among CSWs, one study among IDU inmates, and one among both CSWs and IDUs. The last study was conducted among blood donors who were $\mathrm{HCV}$ seropositive.

\section{HIV-HCV Co-infection Prevalence}

The overall HIV-HCV co-infection prevalence among HIVpositive persons ranged broadly from $1.2 \%$ to $98.5 \%$ in South and Southeast Asia, as shown in Table 2. In six studies testing HIV-positive samples, the HIV-HCV co-infection rate was relatively higher in Indonesia, Nepal and Vietnam, rang-

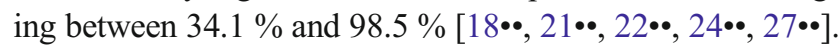
Particularly in Vietnam, it reached $89.8 \%$ and $98.5 \%$ among

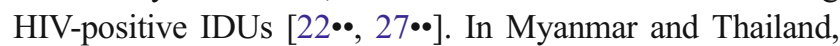
the HIV-HCV co-infection rate among HIV-infected persons was comparatively lower than in Indonesia, Nepal, and Vietnam, $5.3 \%[17 \bullet \bullet]$ and $3.3 \%[29 \bullet \bullet]$ respectively. If the target population was persons with high risk behaviors, such as CSWs in India or IDU inmates in Indonesia, the coinfection prevalence were lower, about $1.2 \sim 4 \%$ [16••, 25••, $26 \bullet \cdot]$. The only study among HCV seropositive blood donors from Nepal published in 2012 showed $5.75 \%$ HIV-HCV co-infection rate $[8 \cdot \bullet]$.

\section{Risk Factors Associated with HIV-HCV Co-infection}

Five cross-sectional studies were conducted among people with obvious high risk behavior, including one study among IDU inmates [26••], one study among HIV-positive IDUs [27••], one study among CSWs [25••], one study among both IDUs and CSWs [22••], and one study among HCV-positive blood donors $[8 \bullet \bullet]$. Five studies estimated the role of risk 


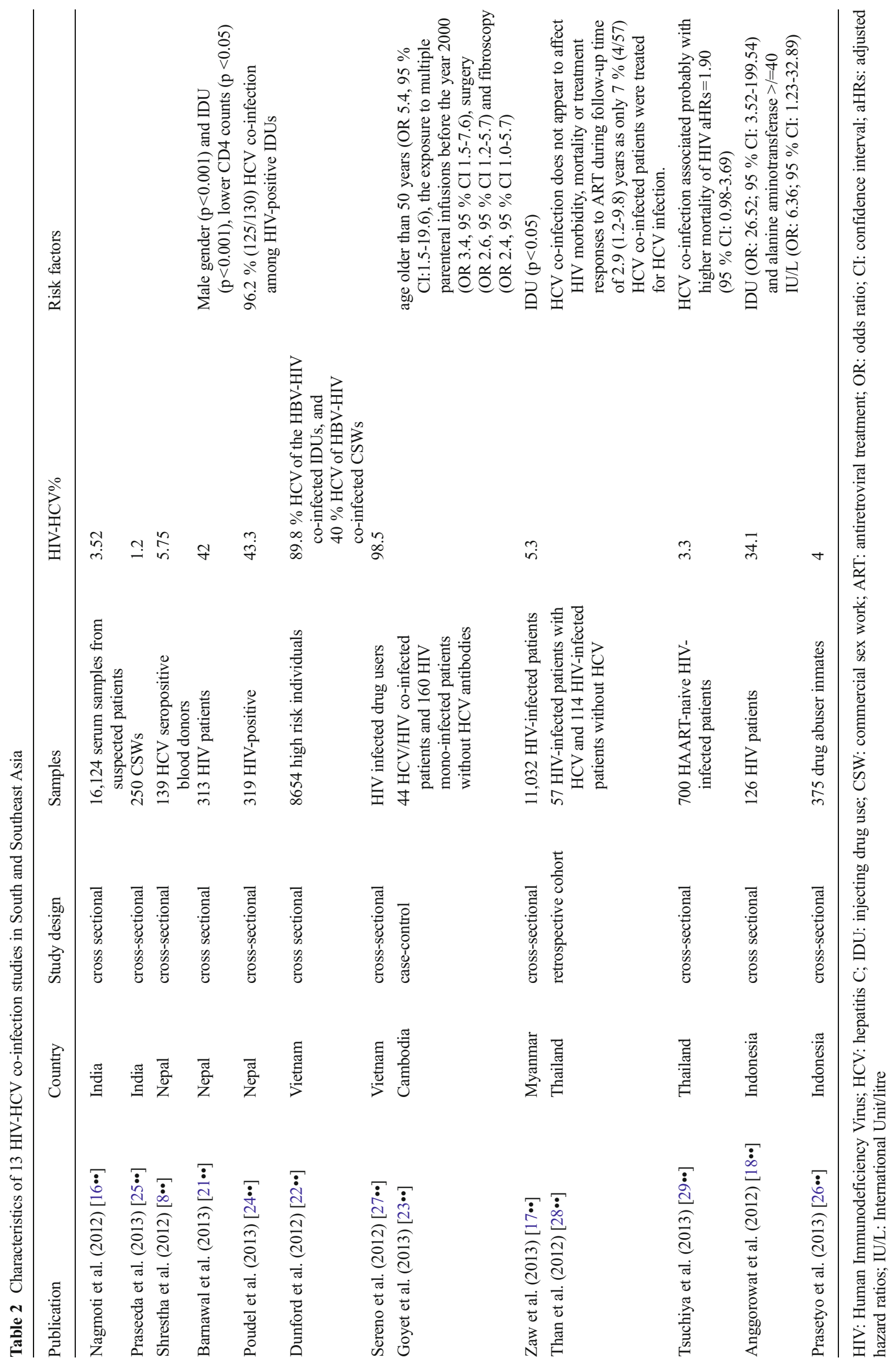


factors associated with HIV-HCV co-infection. Four crosssectional studies strongly indicated IDU as the highest risk factor with a high proportion of co-infection [24••], suggested

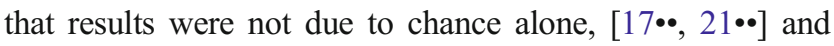
reported strong association in odds ratios [18••]. The only case-control study did not find an association with IDU, rather showing associations with age older than 50 years (OR 5.4, $95 \%$ confidence interval (CI):1.5-19.6), the exposure to multiple parenteral infusions before the year 2000 (OR 3.4, $95 \%$ CI 1.5-7.6), surgery (OR 2.6, $95 \%$ CI 1.2-5.7), and fiber optic scoping (OR 2.4, $95 \%$ CI 1.0-5.7) [23••]. Other risk factors associated with HIV-HCV co-infection were male sex $(\mathrm{p}<$ $0.001)$, lower CD4+ cell counts $(\mathrm{p}<0.05)[21 \bullet \bullet]$, and alanine aminotransferase $>40$ IU/L (OR: 6.36; $95 \%$ CI: $1.2-32.9)[18 \bullet \bullet]$.

Two studies examined the association of HIV-HCV coinfection with other outcomes, but few associations were significant; a probable higher mortality of HIV was noted with $\mathrm{HCV}$ co-infection (adjusted hazard ratios $[\mathrm{aHRs}]=1.90 ; 95 \%$ CI: $0.98-3.7)$ in one cross-sectional study [29••]. One retrospective cohort study suggested that HIV-HCV co-infection had no impact on morbidity, mortality, or treatment responses to ART, as there were HIV patient deaths during 2.9 years of follow-up, although only $7 \%$ of HIV-HCV co-infected patients were treated for HCV infection in the case group [28••]. One study provided HIV-HCV co-infection rate but did not analyze risk factors [16••] (Table 2):

\section{Discussion}

$\mathrm{HIV}$ and $\mathrm{HCV}$ are in completely different virus families, yet both are transmitted through bodily fluids and blood; both viruses can infect patients for years before symptoms are manifested [8••]. Given the attention given to HIV, it is more likely that HCV will be neglected; such neglect can result in irreversible hepatocirrhosis and hepatocarcinoma [1•]. Since 1985, HIV screening has been suggested for the blood supply and for persons at risk, with supportive public policies typically supporting this strategy. In contrast, HCV has commanded far less attention and public policies have lagged the need. We think the current literature does a good job alerting the public as to the seriousness of HCV and HIV$\mathrm{HCV}$ joint infection, promoting screening and treatment policies based on a strategy to improve HCV medical care and prevention.

HIV-HCV co-infection publications suggested wide prevalence variability, from $1.2 \%$ to $98.5 \%$ HIV-HCV coinfection in South and Southeast Asia, notably higher among IDUs (34.1-98.5\%). The multivariable analyses in these cross-sectional studies confirm IDU as the major risk factor in this region, both for HIV-HCV co-infection, and for HIV and $\mathrm{HCV}$ independently. However, the case-control study in
Cambodia suggested multiple parenteral infusions before the year 2000, surgery, and fibroscopy to be the principal risk factors, suggesting diversity between population samples [23••]. The lack of impact of HIV-HCV co-infection on morbidity, mortality or treatment responses to ART of HIV patients in two studies [30,31] clashes with findings from other studies worldwide and may reflect methodological limitations of sample size and length of follow-up.

Most countries in South and Southeast Asia are not yet economically developed and there are strong forces towards increasing high risk behaviors such as IDU and CSW. Basic medical services are insufficient and often very expensive for poor people. Furthermore, HIV prevention and control programs are often weak with inadequate attention and funding support from national and local governments, even though political commitments to Millennium Development Goals (MDGs) have been declared.

The serious epidemics of HIV-HCV co-infection should be emphasized in the prevention and treatment of both diseases in Asia as there was less published research and fewer evidence prevention strategies compared to Western nations. Coinfection may accelerate the clinical progress to both diseases $[13,31-33]$ and treatment success for one disease is undermined when the other disease is neglected. Governments have paid greater attention to HIV prevention and control and typically provide free ARV treatment for HIV, but the high expenses of interferon and ribavirin for $\mathrm{HCV}$ treatment is still a great challenge which prohibits the access of this clinical service, especially in these resource limited settings of South and Southeast countries [28••]. These studies suggest national policies, strategies, and guidelines on HIVHCV co-infection control should be revisited, including other blood-borne infections, such as hepatitis $B$ virus, $G B$ virus $C$

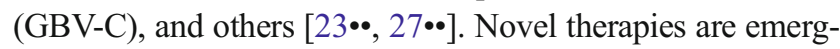
ing quickly for $\mathrm{HCV}$ disease and countries must prepare for the increased opportunities for HCV management that will be emerging.

It is clear that HCV screening should be conducted as soon as an HIV-positive test is confirmed. This permits co-infection to be treated appropriately, quality care provided, and further

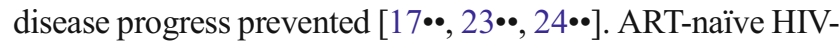
infected patients may have tenofovir-based regimens given preferentially, given its impact on both HIV and HCV, for coinfected persons [29••]. Some unique communities, like prisons, are recommended to pay added attention to screening, prevention, and control of both infections and co-infection among inmates who used or continue to use drugs [26••].

Needle and syringe exchanges, opioid substitution therapy (especially with methadone maintenance or buprenorphine), and condom use are all advisable for persons who use drugs, to modulate risk from body fluid and blood-borne diseases. The sterilization of medical instruments is critical in resource limited settings if disposable syringe or medical consumable 
products are not available [34, 35]. Medical behavior should be monitored strictly to ensure high quality services and lower unprotected exposure to clinical HIV or HCV infections. Proper management of HIV-HCV co-infection is critical and can be highly effective in disease control [36]. Prevention and control strategies should be implemented comprehensively to ensure their maximal effectiveness.

\section{Compliance with Ethics Guidelines}

Conflict of Interest Shaodong Ye, Lin Pang, Xiaochun Wang, and Zhongfu Liu declare that they have no conflict of interest.

Human and Animal Rights and Informed Consent This article does not contain any studies with human or animal subjects performed by any of the authors.

Open Access This article is distributed under the terms of the Creative Commons Attribution License which permits any use, distribution, and reproduction in any medium, provided the original author(s) and the source are credited.

\section{References}

Papers of particular interest, published recently, have been highlighted as:

- Of importance

-. Of major importance

1. World Health Organization. Hepatitis C Fact Sheets. July 2013. Available from: http:/www.who.int/mediacentre/factsheets/fs 164/ en/. This HCV fact sheets provided updated HCV epidemics world widely.

2. Joint United Nations Programme on HIV/AIDS. 2013 UNAIDS Report on the global AIDS epidemic. December 2013. Available from: http:/www.unaids.org/en/media/unaids/contentassets/ documents/epidemiology/2013/gr2013/UNAIDS_Global_Report 2013_en.pdf. This report provided updated HIV epidemics world widely.

3. Barth RE, Huijgen Q, Taljaard J, et al. Hepatitis B/C and HIV in sub-Saharan Africa: an association between highly prevalent infectious diseases. A systematic review and meta-analysis. Int J Infect Dis. 2010;14(12):e1024-31.

4. Zhuang X, Liang Y, Chow EP, et al. HIV and HCV prevalence among entrants to methadone maintenance treatment clinics in China: a systematic review and meta-analysis. BMC Infect Dis. 2012;12:130.

5. Maier I, Wu GY. Hepatitis C and HIV co-infection: a review. World J Gastroenterol. 2002;8(4):577-9.

6. Danta M, Dusheiko GM. Acute HCV in HIV-positive individuals a review. Curr Pharm Des. 2008;14(17):1690-7.

7. Kontorinis N, Agarwal K, Dieterich DT. Treatment of hepatitis C virus in HIV patients: a review. AIDS. 2005;19 Suppl 3:S166-73.

8.• Shrestha AC, Ghimire P, Tiwari BR, et al. Co-infection rate of HIV, HBV and Syphilis among HCV seropositive identified blood donors in Kathmandu, Nepal. Infect Ecol Epidemiol. 2012;2. This study was conducted among $139 \mathrm{HCV}$ seropositive blood donors and the HIV-HCV co-infection rate was $5.75 \%$.
9. Operskalski EA, Kovacs A. HIV/HCV co-infection: pathogenesis, clinical complications, treatment, and new therapeutic technologies. Curr HIV/AIDS Rep. 2011;8(1):12-22.

10. Sulkowski MS. HCV therapy in HIV-infected patients. Liver Int. 2013;33 Suppl 1:63-7.

11. Agdamag DM, Kageyama S, Alesna ET, et al. Rapid spread of hepatitis $\mathrm{C}$ virus among injecting-drug users in the Philippines: implications for HIV epidemics. J Med Virol. 2005;77(2):221-6.

12. Zhao L, Feng Y, Xia XS. The different epidemic and evolution of HCV genotypes. Yi Chuan. 2012;34(6):666-72.

13. Law WP, Duncombe CJ, Mahanontharit A, et al. Impact of viral hepatitis co-infection on response to antiretroviral therapy and HIV disease progression in the HIV-NAT cohort. AIDS. 2004;18(8): 1169-77.

14. Saravanan S, Velu V, Kumarasamy N, et al. Coinfection of hepatitis $\mathrm{B}$ and hepatitis $\mathrm{C}$ virus in HIV-infected patients in south India. World J Gastroenterol. 2007;13(37):5015-20.

15. Ponamgi SP, Rahamathulla S, Kumar YN, et al. Prevalence of hepatitis $\mathrm{C}$ virus (HCV) coinfection in HIV infected individuals in south India and characterization of HCV genotypes. Indian J Med Microbiol. 2009;27(1):12-6.

16.• Nagmoti MB, Patil CS, Jyoti MN, et al. Prevalence of hepatitis C infection in HIV-seropositive individuals in and around Belgaum, south India. Int J STD AIDS. 2012;23(10):e14-5. This study was conducted among 16,124 serum samples from suspected patients and the HIV-HCV co-infection rate was $3.52 \%$.

17.• Zaw SK, Tun ST, Thida A, et al. Prevalence of hepatitis C and B virus among patients infected with HIV: a cross-sectional analysis of a large HIV care programme in Myanmar. Trop Doct. 2013;43(3):113-5. This study was conducted among 11,032 HIVinfected patients and the HIV-HCV co-infection rate was $5.3 \%$.

18.• Anggorowati N, Yano Y, Heriyanto DS, et al. Clinical and virological characteristics of hepatitis B or C virus co-infection with HIV in Indonesian patients. J Med Virol. 2012;84(6):857-65. This study was conducted among $126 \mathrm{HIV}$ patients and the HIV-HCV coinfection rate was $34.1 \%$.

19. Ocama P, Seremba E. Management of HIV and hepatitis C virus infections in resource-limited settings. Curr Opin HIV AIDS. 2011;6(6):539-45.

20. Matthews GV, Dore GJ. HIV and hepatitis C coinfection. J Gastroenterol Hepatol. 2008;23(7 Pt 1):1000-8.

21.• Barnawal SP, Niraula SR, Agrahari AK et al. Human immunodeficiency virus and hepatitis $\mathrm{C}$ virus coinfection in Nepal. Indian $\mathrm{J}$ Gastroenterol. 2013. This study was conducted among 313 HIV patients and the HIV-HCV co-infection rate was $42 \%$.

22.• Dunford L, Carr MJ, Dean J, et al. A multicentre molecular analysis of hepatitis B and blood-borne virus coinfections in Viet Nam. PLoS One. 2012;7(6):e39027. This study was conducted among 8654 high risk individuals. $89.8 \% \mathrm{HCV}$ of the HBV-HIV co-infected IDUs and $40 \% \mathrm{HCV}$ of $\mathrm{HBV}$ HIV co-infected CSWs were found.

23.• Goyet S, Lerolle N, Fournier-Nicolle I, et al. Risk factors for hepatitis C transmission in HIV patients, Hepacam Study, ANRS 12267 Cambodia. AIDS Behav. 2013. This case-control study had $44 \mathrm{HCV} / \mathrm{HIV}$ co-infected patients and $160 \mathrm{HIV}$ mono-infected patients without HCV antibodies, the risk factors associated with co-infection included age older than 50 years, the exposure to multiple parenteral infusions before the year 2000, surgery and fibroscopy.

24.• Poudel KC, Palmer PH, Jimba M, et al. Coinfection of Hepatitis C Virus among HIV-Positive People in the Kathmandu Valley, Nepal. J Int Assoc Provid AIDS Care. 2013. This study was conducted among $319 \mathrm{HIV}$-positive and the HIV-HCV co-infection rate was $43.3 \%$.

25.• Praseeda SD, Anuradha D, Jayanthi SS. A study on the HBV and the $\mathrm{HCV}$ infections in female sex workers and their co-infection 
with HIV. J Clin Diagn Res. 2013;7(2):234-7. This study was conducted among $250 \mathrm{CSW}$ and the HIV-HCV co-infection rate was $1.2 \%$.

26.• Prasetyo AA, Dirgahayu P, Sari Y, et al. Molecular epidemiology of HIV, HBV, HCV, and HTLV-1/2 in drug abuser inmates in central Javan prisons, Indonesia. J Infect Dev Ctries. 2013;7(6):453-67. This study was conducted among 375 drug abuser inmates and the HIV-HCV co-infection rate was $4 \%$.

27.• Sereno L, Mesquita F, Kato M, et al. Epidemiology, responses, and way forward: the silent epidemic of viral hepatitis and HIV coinfection in Vietnam. J Int Assoc Physicians AIDS Care (Chic). 2012;11(5):311-20. This study reviewed data of HIV in Vietnam and the HIV-HCV co-infection rate reached $98.5 \%$ among HIV infected drug users.

28.• Than NN, Sungkanuparph S, Maek-A-Nantawat W, et al. Comparison of clinical outcomes between HIV-infected patients with and without HCV co-infection in a resourcelimited setting. Southeast Asian J Trop Med Public Health. 2012;43(3):646-51. This retrospective cohort study had 57 $\mathrm{HIV}$-infected patients with HCV and $114 \mathrm{HIV}$-infected patients without $H C V$. It found that $H C V$ co-infection did not appear to affect HIV morbidity, mortality or treatment responses to ART.

29.• Tsuchiya N, Pathipvanich P, Rojanawiwat A, et al. Chronic hepatitis $\mathrm{B}$ and $\mathrm{C}$ co-infection increased all-cause mortality in HAART-naive HIV patients in Northern Thailand. Epidemiol Infect. 2013;141(9):1840-8. This study was conducted among 700 HAART-naive HIV-infected patients and the HIV-HCV co-infection rate was $3.3 \%$.

30. Vivithanaporn P, Nelles K, DeBlock L, et al. Hepatitis C virus coinfection increases neurocognitive impairment severity and risk of death in treated HIV/AIDS. J Neurol Sci. 2012;312(1-2):45-51.

31. Salmon-Ceron D, Lewden C, Morlat P, et al. Liver disease as a major cause of death among HIV infected patients: role of hepatitis C and B viruses and alcohol. J Hepatol. 2005;42(6):799-805.

32. Rockstroh JK, Peters L, Grint D, et al. Does hepatitis C viremia or genotype predict the risk of mortality in individuals co-infected with HIV? J Hepatol. 2013;59(2):213-20.

33. Ragni MV, Nalesnik MA, Schillo R, Dang Q. Highly active antiretroviral therapy improves ESLD-free survival in HIV-HCV coinfection. Haemophilia. 2009;15(2):552-8.

34. Szaflik J, Bełzecka-Majszyk A. Patients with HBV, HCV and HIV antigens-medical management, surgical procedures, equipment sterilization principles. Klin Oczna. 2003;105(6):448-52.

35. Ouzan D. Risk of transmission of hepatitis C through endoscopy of the digestive tract. Presse Med. 1999;28(20):1091-4.

36. Santana JL, Rodríguez-Medina JR, Rodríguez-Orengo JF. Clinical challenges and controversies in the management of $\mathrm{HIV} / \mathrm{HCV}$-coinfected individuals. $\mathrm{P} \mathrm{R}$ Health Sci J. 2004;23(2 Suppl):35-40. 